\title{
Does the World Health Organization criterion adequately define glaucoma blindness?
}

\author{
This article was published in the following Dove Press journal: \\ Clinical Ophthalmology \\ 2 March 2017 \\ Number of times this article has been viewed
}

\section{P Mokhles \\ JSAG Schouten \\ HJM Beckers \\ CAB Webers}

University Eye Clinic Maastricht, Maastricht, the Netherlands
Correspondence: P Mokhles Maastricht University Medical Center, University Eye Clinic Maastricht, P.Debyelaan 25, $6229 \mathrm{HX}$, the Netherlands

$\mathrm{Tel}+3$ I 433875346

Fax +3I 433875343

Email palwasha.mokhles@mumc.nl
Purpose: Blindness in glaucoma is difficult to assess with merely the use of the current World Health Organization (WHO) definition (a visual field restricted to $10^{\circ}$ in a radius around central fixation), as this criterion does not cover other types of visual field loss that are encountered in clinical practice and also depict blindness. In this study, a 5-point ordinal scale was developed for the assessment of common visual field defect patterns, with the purpose of comparing blindness as outcome to the findings with the WHO criterion when applied to the same visual fields. The scores with the two methods were compared between two ophthalmologists. In addition, the variability between these assessors in assessing the different visual field types was determined.

Methods: Two glaucoma specialists randomly assessed a sample of 423 visual fields from 77 glaucoma patients, stripped of all indices and masked for all patient variables. They applied the WHO criterion and a 5-point ordinal scale to all visual fields for the probability of blindness.

Results: The WHO criterion was mostly found applicable and in good agreement for both assessors to visual fields depicting central island of vision or a temporal crescent. The percentage of blindness scores was higher when using the ordinal scale, $21.7 \%$ and $19.6 \%$ for assessors A and B, respectively, versus $14.4 \%$ and $11.3 \%$ for the WHO criterion. However, Kappa was lower, 0.71 versus 0.78 for WHO.

Conclusions: The WHO criterion is strictly applied and shows good agreement between assessors; however, blindness does not always fit this criterion. More visual fields are labeled as blind when a less stringent criterion is used, but this leads to more interobserver variability. A new criterion that describes the extent, location, and depth of visual field defects together with their consequence for the patient's quality of life is needed for the classification of glaucoma blindness.

Keywords: glaucoma, blindness, visual fields, World Health Organization

\section{Introduction}

From a societal perspective, it is important to establish the degree of visual impairment and blindness in glaucoma patients. To reach this goal, a classification based on the location and extensiveness of glaucomatous visual field loss is needed. Currently, visual disability is defined with the strict World Health Organization (WHO) criterion that defines blindness as a visual acuity $<0.05$ and/or a visual field $<10^{\circ}$ in a radius around the central fixation in the better eye. ${ }^{1}$ Unfortunately, there are many examples of visual field defects where the definition of the WHO is not conclusive or where visual field loss does not properly fit this criterion (eg, an isolated scotoma within $10^{\circ}$ or an altitudinal defect). Glaucoma specialists, based on their clinical experience, may regard these examples as extremely invalidating. Therefore, the WHO definition does not seem to be the proper tool to assess glaucoma blindness in daily clinical practice. 
A broader classification than the WHO definition will label more patients as blind or visually impaired (eg, in patients with an altitudinal defect in the lower hemifield), and because of this, the reported prevalence of registered glaucoma blindness or visual impairment would be higher. It is important to register blind or visually impaired patients appropriately to allow them to claim supportive care. A new, clearly defined and a comprehensive criterion is needed to ensure comparability and cover different types of visual field defects.

We initiated this study as a first step toward finding an alternative and additional criterion to assess blindness, based on the location and extensiveness of visual field defects. We developed a 5-point ordinal scale for the assessment of common visual field defect patterns, with the purpose of comparing blindness as outcome to the findings of the WHO criterion when applied to the same visual fields. First, we applied the WHO criterion to a set of visual fields to assess the prevalence of blindness and the agreement of the scores between two glaucoma specialists. Subsequently, we investigated the prevalence of blindness and the agreement with the alternative and less strict 5 -point ordinal scale. Finally, the variability between the two glaucoma specialists in assessing the different visual field types was examined.

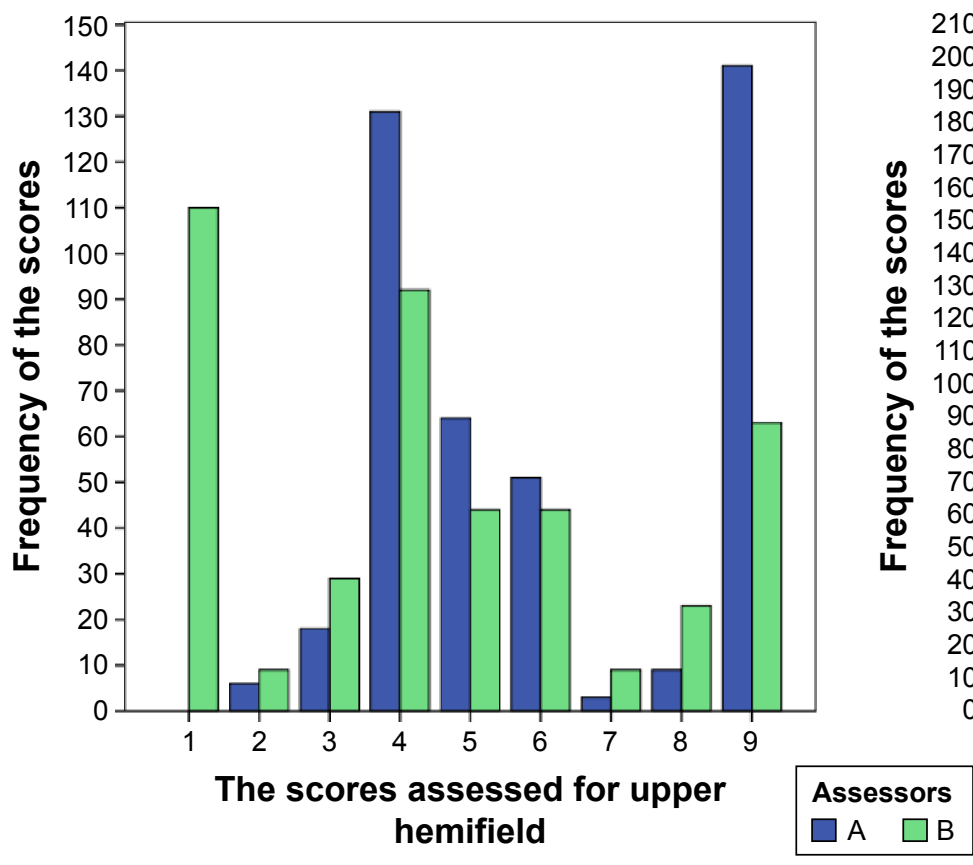

\section{Materials and methods}

This study was conducted in accordance with the tenets of the Declaration of Helsinki and was approved by the Medical Ethical Committee in Maastricht University Medical Center (Maastricht, the Netherlands). Patient consent has been obtained.

\section{Study design}

Interobserver and prevalence study.

\section{Study population}

A sample from a cohort of 3,883 glaucoma (suspect) patients who participated in the DURING study (DUtch Research project on treatment outcome IN Glaucoma patients) in nine hospitals (academic, teaching, and nonteaching) between 2001 and $2004^{2}$ was included in this study.

\section{Assessment of visual field type and blindness}

Visual fields from the Humphrey Visual Field Analyzer (HFA; Carl Zeiss Meditec, Dublin, CA, USA) or an analog instrument (Goldmann or Peritest [Rodenstock, Munich, Germany]) were used. The last most recent visual fields were chosen for this study, with a maximum of three. First, the visual field tests from all patients were numbered randomly. Subsequently,

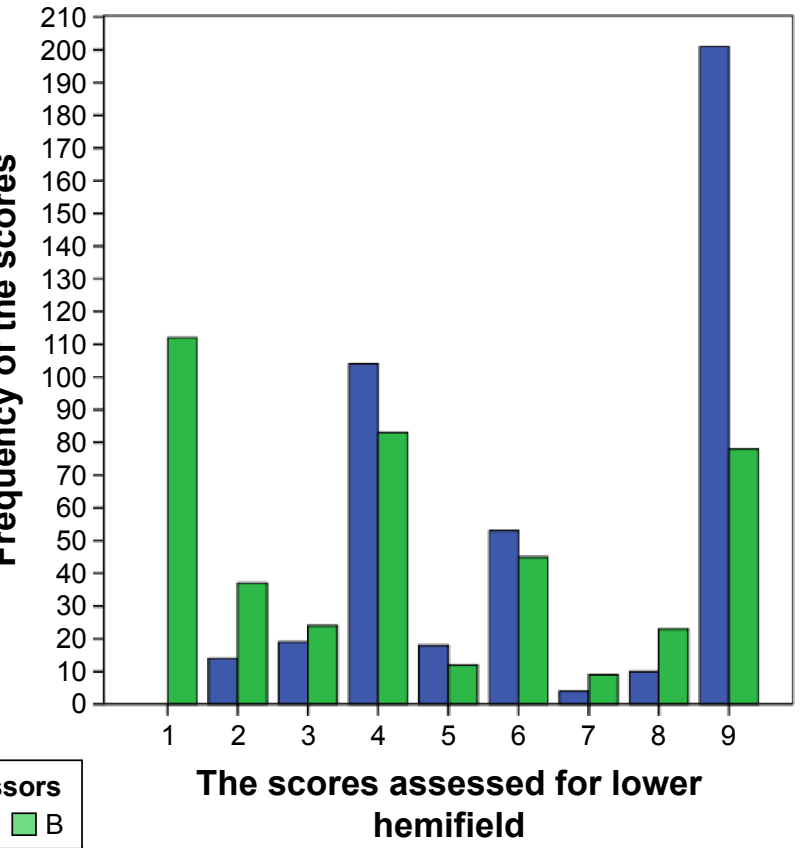

Figure I The variability in the scores of the type of visual field between assessor A and B for upper and lower hemifield.

Notes: The x-axis represents the scores that are given for upper and lower hemifield; I) overall sensitivity loss, 2) nasal step, 3) para-central scotoma, 4) arcuate scotoma, 5) altitudinal defect, 6) central island of vision, 7) temporal crescent, 8) hemianopia, and 9) no visual field defect present. The $y$-axis represents the amount of times each score has been given. The colors blue and green represent assessors $\mathrm{A}$ and $\mathrm{B}$, respectively. 
all visual fields were anonymized and stripped of all indices. Thus, the patients' name and date of birth, right or left eye, the date of the visual field test and all other indices, and printed data were removed from the visual fields, except for the gray scale print. Similar actions were taken for Goldmann and Peritest visual fields. Finally, all tests were rearranged in a random order with each eye and each patient mixed through the whole set of the visual fields. As a result, only monocular blindness was assessed. Furthermore, the definition of visual field type was not agreed on or discussed between the assessors beforehand. The assessors, two glaucoma specialists ( $\mathrm{A}$ and $\mathrm{B}$ ), were presented with this last version of the anonymized visual fields, with only the type of the test (HFA 30-2, HFA 24-2, HFA 10-2, and Goldmann or Peritest) and the random number of the visual field visible for them. The assessors scored each visual field for each category of blindness in a separate file provided with these visual fields.

The assessors assessed blindness according to the WHO visual field criterion, that is, visual field $<10^{\circ}$ in a radius around central fixation. They also assessed blindness according to a 5-point ordinal scale consisting of the following categories: blind, most probably blind, probably blind, probably not blind, and not blind. And finally, the type of visual field loss was assessed according to location and extensiveness, and rated as overall sensitivity loss, nasal step, para-central scotoma, arcuate scotoma, altitudinal defect, central island of vision, temporal crescent, hemianopia, and no visual field defect present. Upper and lower hemifield were rated separately, because of the variable spectrum of visual field defects, which can be present in one visual field examination, for example, presence of a para-central scotoma in the upper hemifield and an altitudinal defect in the lower hemifield, which cannot be scored as one entity.

\section{Data analyses}

The percentages of the scores given by each assessor for the two criteria and the agreement of these scores between the assessors were analyzed using IBM SPSS (version 23.0; IBM Corp., Armonk, NY, USA). The agreement was calculated using the Kappa statistic. This measures the agreement between two raters and is described as follows: $<0.00$ poor agreement, $0.00-0.20$ slight agreement, $0.21-0.40$ fair agreement, $0.41-0.60$ moderate agreement, $0.61-0.80$ substantial agreement, and 0.81-1.00 almost perfect agreement. ${ }^{3}$ First, the percentage of blindness according to the $\mathrm{WHO}$ criterion was calculated per assessor. Next, the agreement, by means of Kappa, was calculated and this was followed by the calculation of the percentage of blindness per visual field type.
The same procedure was used for the rating of blindness on the 5-point ordinal scale. For this analysis, the categories of the 5-point ordinal scale were dichotomized: the items "blind", "most probably blind", and "probably blind" were combined to the category "blind" and the items "probably not blind" and "not blind" to "not blind". Furthermore, we assessed the number of similarly rated visual fields (with regard to the type of visual field) and the variability between the assessors in visual field type scores, to identify possible sources of disagreement in the ratings of blindness.

In addition, an analysis in which the two ophthalmologists graded the nine visual field types according to the ordinal scale was performed, to investigate if the outcomes would yield comparable results.

\section{Results}

From the total cohort of 3,883 patients from the DURING study (described earlier), we included a random sample of 77 patients with 423 visual fields from one university hospital for the analyses.

\section{Assessment and variability using the WHO criterion}

Using the WHO criterion, assessor A rated $14.4 \%$ of the visual fields as blind and assessor B 11.3\%, with a kappa of 0.78 (Table 1). The visual fields central island of vision and temporal crescent were mostly assessed as blind according to this criterion. The variability between the assessors was found in cases where visual fields depicted altitudinal defects, central islands of vision, and para-central scotomas (not shown in the table).

\section{Assessment and variability using a 5-point ordinal scale}

A higher percentage of blindness was found when the ordinal scale was used as an alternative for the WHO criterion; $21.7 \%$ and $19.6 \%$ of the visual fields were rated as blind according to assessors $\mathrm{A}$ and $\mathrm{B}$, respectively, but with a lower kappa of 0.71 (Table 2). Central islands of vision and

Table I Frequency of blindness according to the $\mathrm{WHO}$ criterion $(\mathrm{N}=423$ visual fields)

\begin{tabular}{lllll}
\hline & $\begin{array}{l}\text { Assessor A, Assessor B, Kappa } \\
\text { N (\%) }\end{array}$ & $\begin{array}{l}\text { Percentage } \\
\text { N (\%) } \\
\text { agreement }\end{array}$ \\
\hline $\begin{array}{l}\text { WHO criterion } \\
\text { score }\end{array}$ & & & \\
$\begin{array}{l}\text { Blind } \\
\text { Not blind }\end{array}$ & $61(14.4)$ & $48(11.3)$ & 0.78 & 95.0 \\
\hline
\end{tabular}

Note: ${ }^{\mathrm{B}}$ Blind $=$ visual field $<10^{\circ}$ in a radius around central fixation. Abbreviation: WHO, World Health Organization. 
Table 2 Frequency of blindness according to the 5-point ordinal scale, dichotomized ( $\mathrm{N}=423$ visual fields)

\begin{tabular}{|c|c|c|c|c|}
\hline & Assessor $\mathrm{A}$ & Assessor B & Kappa & Percentage \\
\hline & $\mathbf{N}(\%)$ & N (\%) & & agreement \\
\hline \multicolumn{5}{|c|}{$\begin{array}{l}\text { 5-Point ordinal scale, } \\
\text { dichotomized }\end{array}$} \\
\hline Blind $^{a}$ & $92(21.7)$ & $83(19.6)$ & 0.71 & 90.3 \\
\hline Not blind ${ }^{b}$ & 331 (78.3) & $340(80.4)$ & & \\
\hline
\end{tabular}

Notes: "Blind = the score "blind", "most probably blind", and "probably blind" according to the 5-point ordinal scale; "Not blind = "probably not blind" and "not blind" according to the 5-point ordinal scale.

temporal crescents were mostly assessed as blind according to the ordinal scale as well. However, this scale showed more variability between the assessors, which was present in the categories arcuate scotoma, altitudinal defect, para-central scotoma, and hemianopia (not shown in the table).

\section{Interobserver variability in visual fields with the same score for the type of defect}

A closer look at the interobserver variability revealed that even when visual fields were given the same rating by the two assessors, the agreement on blindness with the ordinal scale was still lower (kappa 0.72) than with the WHO criterion (kappa 0.91; Table 3). The assessment of visual field type showed imperfect overlap between the assessors. The main discrepancies were seen between the categories central island of vision, altitudinal defect, and arcuate scotoma for upper and lower hemifield.

\section{Additional analysis on the assessment of type of visual field with the 5-point ordinal scale}

An additional analysis in which the two ophthalmologists graded the nine visual field types according to the ordinal

Table 3 Frequency of blindness according to the WHO criterion and 5 -point ordinal scale, when visual field type was rated similarly ( $N=224$ visual fields)

\begin{tabular}{|c|c|c|c|c|}
\hline & $\begin{array}{l}\text { Assessor A, } \\
\mathbf{N}(\%)\end{array}$ & $\begin{array}{l}\text { Assessor B, } \\
\mathbf{N}(\%)\end{array}$ & Kappa & $\begin{array}{l}\text { Percentage } \\
\text { agreement }\end{array}$ \\
\hline \multicolumn{5}{|c|}{$\begin{array}{l}\text { WHO criterion } \\
\text { score }\end{array}$} \\
\hline Blind ${ }^{a}$ & $4 \mid(18.3)$ & 35 (I5.6) & 0.91 & 97.3 \\
\hline Not blind & I83 (8I.7) & I89 (84.4) & & \\
\hline \multicolumn{5}{|c|}{$\begin{array}{l}\text { 5-Point ordinal } \\
\text { scale, dichotomized }\end{array}$} \\
\hline Blind ${ }^{b}$ & $64(28.6)$ & $59(26.3)$ & 0.72 & 97.5 \\
\hline Not blind ${ }^{c}$ & 160 (7I.4) & 165 (73.7) & & \\
\hline
\end{tabular}

Notes: ${ }^{\mathrm{B}}$ Blind $=$ visual field $<10^{\circ}$ in a radius around central fixation; ${ }^{\mathrm{b}}$ Blind $=$ the score "blind", "most probably blind", and "probably blind" according to the fivepoint ordinal scale; "Not blind = "probably not blind" and "not blind" according to the five-point ordinal scale.

Abbreviation: WHO, World Health Organization. scale showed almost similar results and almost no variability between the assessors, except for the type of visual field "hemianopia"; assessor A scored this as "probably blind" and assessor B scored this as "not blind" (Table 4). This additional analysis shows that the ophthalmologists almost always agree about the relation of the type of visual field to blindness but differ in the interpretation of the visual field.

\section{Discussion}

This study shows that the WHO visual field criterion for blindness can be stringently applied with a good agreement between two glaucoma specialists. However, this is mainly true for straight cases, with visual fields depicting a central island of vision or a temporal crescent, for which this criterion probably was initially formulated, that is, defining blindness as a visual field $<10^{\circ}$ in a radius around central fixation. Unfortunately, there are many variations in visual fields that do not fit this criterion properly (eg, altitudinal defects in the lower hemifield), which are known to have an impact on quality of life ${ }^{4,5}$ and are often rated as blindness by ophthalmologists. In our study, these were the cases with a larger variability between the two assessors. This inapplicability of the WHO criterion probably leads to underreporting of blindness. To determine if the agreement between the WHO criterion and the 5-point ordinal scale could be improved, an additional analysis was performed. In this analysis, only the items "blind" and "most probably blind" were included in the category "blind" of the 5-point ordinal scale (ie, excluding the item "probably blind") and this showed a similar kappa of 0.70 as compared to the one mentioned in the results earlier. Thus, even after creating a more specified category "blind", the difference in agreement of assessing blindness between the WHO criterion and the 5-point ordinal scale remained the same.

We made an effort to solve this problem by designing a 5-point ordinal scale of which we thought it could provide the ophthalmologist with more options to assess blindness according to their expertise. Indeed, we found more cases of blindness with this method; however, at the expense of more interobserver variability. The variability with the 5-point ordinal scale was probably due to the fact that this classification scale allows the ophthalmologist's more personal interpretation to decide which visual field type is supposed to cause blindness. The two glaucoma specialists determined the type of visual field as well and this showed inconsistencies between them. The discrepancies were mostly seen in the defects central island of vision, altitudinal defect, and arcuate scotoma. The variability was due to a difference in the interpretation of absolute and relative scotomas (in part 
Table 4 Assessment of type of visual field (defect) with the 5-point ordinal scale

\begin{tabular}{|c|c|c|c|c|}
\hline \multirow[t]{3}{*}{ Type of visual field (defect) } & \multicolumn{4}{|c|}{ 5-Point ordinal scale } \\
\hline & Assessor A & Assessor B & Assessor A & Assessor B \\
\hline & Upper hemifield & Upper hemifield & Lower hemifield & Lower hemifield \\
\hline Overall sensitivity loss & Not blind & Not blind & Not blind & Not blind \\
\hline Nasal step & Not blind & Not blind & Not blind & Not blind \\
\hline Para-central scotoma & Probably not blind & Probably not blind & Probably not blind & Probably not blind \\
\hline Arcuate scotoma & Probably not blind & Probably not blind & Probably not blind & Probably not blind \\
\hline Altitudinal defect & Probably not blind & Probably not blind & Probably blind & Probably blind \\
\hline Central island of vision & Most probably blind & Most probably blind & Most probably blind & Most probably blind \\
\hline Temporal crescent & Blind & Blind & Blind & Blind \\
\hline Hemianopia & Probably blind & Not blind & Probably blind & Not blind \\
\hline No visual field defect present & Not blind & Not blind & Not blind & Not blind \\
\hline
\end{tabular}

because of the gray scale quality of the visual fields) and a difference in the interpretation of the visual fields (before starting the assessment, the assessors did not discuss or agree on the desired definition of the visual field type). Certain types of visual fields caused the difference in interpretation as well; for example, one assessor interpreted an extensive arcuate scotoma as an altitudinal defect, while the other assessor interpreted it as an arcuate scotoma, or, for example, an extensive para-central scotoma being interpreted as an arcuate scotoma. This is to a large part also a semantic issue, which was easily resolved after a discussion on the naming of visual field defects.

Adequate assessment of the type of visual field defect is essential, because the impact of visual field loss on quality of life does not only depend on the severity or extent of it but also on the location of the defect. For example, studies have shown that visual field loss of the lower hemifield is associated with a poorer quality of life compared to visual field loss of the upper hemifield. ${ }^{4-7}$ This is because lower hemifield obtains the main part of the visual information that is used in the lower limb movements. ${ }^{4-6}$

A majority of patients with loss of vision will in the end lose their driver's license, as the legal requirements for it are strict. The European/Dutch requirements for Group 1 license entitlement entail the following: a visual field of at least $120^{\circ}$ horizontally, which must extend $50^{\circ}$ to the left and the right; the vertical visual field should be at least $20^{\circ}$ above and below; absence of visual field defects within a radius of $20^{\circ}$ from the central area. For Group 2 license entitlement (lorries, busses, and minibuses), these conditions are even stricter. ${ }^{8}$ Thus, most glaucoma patients, even before advanced visual field loss develops, will not meet these requirements. Losing one's driver's license has a great impact on patients, and it leads to immobility, social dependence, and a greater risk for developing depressive symptoms. ${ }^{5,7,9-12}$ These consequences of visual impairment not only have an impact on patients' quality of life (eg, difficulty with walking, reading, balance, and driving) but also on patients' caregivers and society at large. ${ }^{5,9-13}$

A strength of the current study is the anonymization and "stripping" of the visual fields. By anonymizing and stripping of all indices before assessment of the visual fields, the assessors were masked for all relevant characteristics of test and patient. This ensured that the assessors were not biased in their judgment by information visible on the regular visual field examination, such as the global indices mean deviation (MD) and visual field index (VFI; inconsistencies between these parameters and the visual field defects could affect the judgment of blindness), patients' name (the assessors could recognize their own patients), separate and independent assessment of the right eye and left eye (assessors could be biased in their judgment if they would know that one eye had more extensive visual field loss than the other eye, considering the effect of bilateral visual impairment or loss), and the date of the visual field examination (assessors could recognize the order of the visual field examinations, considering the progression of the disease). Another strength of this study is the inclusion of a large amount of visual fields for the assessment of blindness. Furthermore, this is the first study illustrating the difficulty with the WHO criterion and illustrating the comparability of it to another criterion.

A limitation of this study is that we could not mask the assessors for the type of visual field examination; this could have influenced the assessment, as a 10-2 visual field examination would probably suggest a more advanced visual field loss compared with a 30-2 visual field. However, in using the WHO criterion, one needs to know which visual field is presented, as blindness is defined as a visual field $<10^{\circ}$ in a radius around central fixation. Another limitation is that there were only two assessors. For future studies, it would be preferable to include more assessors of preferably more 
centers to study causes of variability and to determine what will be needed to further improve a criterion. Furthermore, a number of visual fields were of a rather poor quality, which made the interpretation of, for example, a relative and an absolute scotoma more difficult. Furthermore, we have not randomized the order of the assessment of visual fields with the WHO and ordinal scale, that is, first assess visual fields with the ordinal scale, rearrange all the visual fields, and then assess it again with the WHO criterion. Nevertheless, the results are most probably not biased as there is still disagreement between the assessors and we assume that if we had randomized, then the disagreement between the assessors probably would even be higher.

As described in the "Materials and methods" section, we intended to include three visual fields for this study. This is because the current study is part of an ongoing larger study (DURING study, described earlier) in which three most recent visual fields before last visit have been selected to have a proper assessment of the amount of visual field loss. In that same set of patient data (acquired from the DURING study), we performed the current study. However, not all patients had three visual fields available, which indicates the 423 visual fields that were assessed and not 462 as would be expected.

To our knowledge, there is one study, by Guerin et al, ${ }^{14}$ that investigated the agreement between ophthalmologists in assessing visual impairment in glaucoma patients. They showed that the intra- and interobserver agreement were very poor between ophthalmologists with regard to eligibility for visual impairment registration. Other studies showed that a majority of blind (glaucoma) patients are not registered properly; that is, due to subjective assessment, blindness is not assessed properly, which leads to underreporting of visual impairment and blindness. ${ }^{15-17}$ These studies reported different causes for underreporting of visual disability, among which different criteria for defining visual impairment and blindness (eg, USA, WHO, and driving eligibility requirements).

Besides the criteria mentioned earlier, there are other criteria that could be used to define blindness and have been used in the past, such as MD and VFI. ${ }^{18,19}$ However, defining blindness with only these parameters would not be an appropriate method either, as the MD distribution does not give any clue of the location of the defect, which has an important value. And in fact, it has been reported that considering only the MD, results in losing information on visual loss, such as in patients with defects in the lower hemifield. ${ }^{7}$ Nevertheless, it has been shown previously that progressive visual field loss (in terms of MD) is associated with loss in health-related quality of life. ${ }^{6}$ Thus, the visual field parameters should be used in assessing blindness, but supplemented with additional characteristics (eg, location and severity of the defect).

The most important aspect that our study wants to emphasize is that it is not easy to find adequate tools for ophthalmologists to define visual disability within the scope of our current visual field test strategies. However, it is clear that there remains an unmet need for an additional and a better criterion.

We need an appropriate criterion because the lack of this can have major implications for individual glaucoma patients. There may be legal or work-related issues, such as losing one's driving license. For example, according to Dutch/European requirements for driver's license (described earlier), patients who do not fulfill the WHO criterion and are defined as "not blind" (a visual field $>10^{\circ}$ ) but do have a visual field of $<20^{\circ}$ around central area are still not allowed to drive. Another example is that ophthalmologists need to correctly select patients who are in need of low vision aid or supportive care offered by health care providers, because this can improve the patients' quality of life further.

In developing a new criterion for glaucoma blindness, one has to consider some important parameters, which also have been mentioned in the literature, but up to now still have not been incorporated in the definition of blindness that is used by physicians in daily practice. For instance, there is a tendency of using only monocular visual field loss, whereas binocular visual loss gives a better understanding of the impact of visual loss on the quality of life. It also gives a better view on functioning in daily life activities, as in some cases, visual field loss seams to appear worse in monocular assessment compared with binocular assessment. ${ }^{13,20,21}$ Another parameter would be the driver's license eligibility requirements, as losing this has an important impact on patients quality of life as mentioned earlier..$^{5,7-12}$ Parameters that indicate the quality of life on the basis of questionnaires should be used as well. The location of the defect is also of significance. It matters, for example, if a patient has a visual field loss in the upper hemifield (interferes with near activities like reading) or lower hemifield (vision used in mobility), as visual field loss in the lower hemifield is more disabling than in the upper hemifield..$^{6,13}$

Considering all this, we propose a revised version of the ordinal scale used in the current study to make this scale more objective and comparable, and to include parameters important for assessing the impact of visual field loss. We recommend the following: implement the type of visual field defect (ie, location of the defect) in the ordinal scale; for example, 
"blind" is temporal crescent, "most probably blind" includes central island of vision, "probably blind" contains defects in the lower hemifield, "probably not blind" includes arcuate scotoma and para-central scotoma, and "not blind" includes overall sensitivity loss and nasal step. Subsequently, each category and the corresponding type of defect should include a definition, for example, what constitutes blindness and central island of vision. Furthermore, it is also important to include the following parameters: 1) parameters that indicate the quality of life (assessed through questionnaires), 2) visual field indices like MD and VFI, 3) measurement of monocular and binocular visual field loss, and 4) driver's license eligibility requirements.

To conclude, the WHO criterion is strict and can be applied stringently causing good interobserver agreement. However, they also lead to underregistration of blindness. A classification as chosen in the present study registers more blind patients but at the cost of a lower interobserver agreement. Although the WHO already extended the definition of blindness from complete lack of light perception toward its present definition, we need to move forward to further widen the definition with an additional and an alternative criterion as mentioned earlier. We have to implement what is already known to be important factors in glaucoma blindness, to the new more broad criterion. Thus, in case of glaucoma patients, the size, the location, and the depth of the visual field defect, visual field indices, monocular and binocular visual loss, quality of life, and other parameters, such as evidencebased driving eligibility requirements, should be included.

\section{Acknowledgment}

All authors have made direct contributions to the intellectual content of the article.

\section{Disclosure}

CABW received nonfinancial support from Alcon and grants and/or personal fees from Alcon, Allergan, Santen, Merck, Sharp, and Dohme. HJMB received nonfinancial support and grants and/or personal fees from Alcon, Allergan, InnFocus, Santen, MSD, and Pfizer. JSAGS received nonfinancial support from Novartis and Sanofi. PM reports no conflicts of interest in this work.

\section{References}

1. who.int [homepage on the internet]: WHO ICD. Change the definition of blindness (ICD 10th revision 1st and 2nd edition). Available from: http://www.who.int/blindness/Change $\% 20$ the $\% 20$ Definition $\% 20$ of $\% 20$ Blindness.pdf?ua=1. Accessed March 1, 2015.
2. van der Valk R, Webers CAB, Hendrikse F, de Vogel SC, Prins MH, Schouten JSAG. Predicting intraocular pressure change before initiating therapy: timolol versus latanoprost. Acta Ophthalmologica. 2008; 86(4):415-418.

3. Landis JR, Koch GC. The measurement of observer agreement for categorical data. Biometrics. 1977;33(1):159-174.

4. Black AA, Wood JM, Lovie-Kitchin JE. Inferior visual field reductions are associated with poorer functional status among older adults with glaucoma. Ophthalmic Physiol Opt. 2011;31(3):283-291.

5. Cheng HC, Guo CY, Chen MJ, Ko YC, Huang N, Liu CJL. Patientreported vision-related quality of life differences between superior and inferior hemifield visual field defects in primary open-angle glaucoma. Jama Ophthalmol. 2015;133(3):269-275.

6. van Gestel A, Webers CAB, Beckers HJM, et al. The relationship between visual field loss in glaucoma and health-related quality-of-life. Eye. 2010;24(12):1759-1769.

7. Crabb DP. A view on glaucoma - are we seeing it clearly? Eye (Lond). 2016;30(2):304-313.

8. gov.uk [homepage on the internet]: For medical practitioners. At a glance guide to the current medical standards of fitness to drive (November 2014 edition including August 2015 amendments). Available from: https://www.gov.uk/government/publications/assessing-fitness-todrive-a-guide-for-medical-professionals (the 2016 version). Accessed August 1, 2015.

9. McKean-Cowdin R, Varma R, Wu J, Hays RD, Azen SP; the Los Angeles Latino Eye Study Group. Severity of visual field loss and healthrelated quality of life. Am J Ophthalmol. 2007;143(6):1013-1023.

10. Haymes SA, LeBlanc RP, Nicolela MT, Chiasson LA, Chauhan BC. Glaucoma and on-road driving performance. Invest Ophthalmol Vis Sci. 2008;49(7):3035-3041.

11. Owen VMF, Crabb DP, White ET, Viswanathan AC, Garway-Heath DF, Hitchings RA. Glaucoma and fitness to drive: Using binocular visual fields to predict a milestone to blindness. Invest Ophthalmol Vis Sci. 2008;49(6):2449-2455.

12. van Landingham SW, Hochberg C, Massof RW, Chan E, Friedman DS, Ramulu PY. Driving patterns in older adults with glaucoma. $B M C$ Ophthalmol. 2013;13:4.

13. Hu S, Smith ND, Saunders LJ, Crabb DP. Patterns of binocular visual field loss derived from large-scale patient data from Glaucoma clinics. Ophthalmology. 2015;122(12):2399-2406.

14. Guerin E, Bouliotis G, King A. Visual impairment registration: evaluation of agreement among ophthalmologists. Eye. 2014;28(7):808-813.

15. Barry RJ, Murray PI. Unregistered visual impairment: is registration a failing system? Br J Ophthalmol. 2005;89(8):995-998.

16. King AJW, Reddy A, Thompson JR, Rosenthal AR. The rates of blindness and of partial sight registration in glaucoma patients. Eye. 2000; 14(pt 4):613-619.

17. Robinson R, Deutsch J, Jones HS, et al. Unrecognized and unregistered visual impairment. Br J Ophthalmol. 1994;78(10):736-740.

18. Ernest PJG, Busch MJWM, Webers CAB, et al. Prevalence of endof-life visual impairment in patients followed for glaucoma. Acta Ophthalmol. 2013;91(8):738-743.

19. Rao HL, Kumar AU, Babu JG, Senthil S, Garudadri CS. Relationship between severity of visual field loss at presentation and rate of visual field progression in glaucoma. Ophthalmology. 2011;118(2):249-253.

20. Saunders LJ, Russell RA, Crabb DP. Practical landmarks for visual field disability in glaucoma. Br J Ophthalmol. 2012;96(9):1185-1189.

21. Crabb DP, Viswanathan AC. Integrated visual fields: a new approach to measuring the binocular field of view and visual disability. Graefes Arch Clin Exp Ophthalmol. 2005;243(3):210-216. 


\section{Publish your work in this journal}

Clinical Ophthalmology is an international, peer-reviewed journal covering all subspecialties within ophthalmology. Key topics include: Optometry; Visual science; Pharmacology and drug therapy in eye diseases; Basic Sciences; Primary and Secondary eye care; Patient Safety and Quality of Care Improvements. This journal is indexed on

Submit your manuscript here: http://www.dovepress.com/clinical-ophthalmology-journal
PubMed Central and CAS, and is the official journal of The Society of Clinical Ophthalmology (SCO). The manuscript management system is completely online and includes a very quick and fair peer-review system, which is all easy to use. Visit http://www.dovepress.com/ testimonials.php to read real quotes from published authors. 